PRESENTATION 2.3.1

N91-28229

COMPOSITE MATERIAL SYSTEMS FOR HYDROGEN MANAGEMENT 



\title{
COMPOSITE MATERIAL SYSTEMS FOR HYDROGEN MANAGEMENT
}

\author{
R.N. Pangborn and R.A. Queeney \\ Dept. of Engineering Science and Mechanics
}

\section{Research Objectives and Impact on Propulsion}

The task of managing hydrogen entry into elevated temperature structural materials employed in turbomachinery is a critical engineering area for propulsion systems employing hydrogen or decomposable hydrocarbons as fuel. Extant structural materials, such as the Inconel series, are embrittled by the ingress of hydrogen in service, leading to a loss of endurance and general deterioration of load-bearing dependability. Although the development of hydrogen-insensitive material systems is an obvious engineering option, to date insensitive systems cannot meet the timetemperature-loading service extremes encountered. A short-term approach that is both teasible and technologically sound is the development and employment of hydrogen barrier coatings.

The present project is concerned with developing, analyzing, and physically testing laminate composite hydrogen barrier systems, employing Inconel 718 as the structural material to be protected. Barrier systems will include all metallic, metallic-to-ceramic, and, eventually, metallic/ceramic composites as the lamellae. Since space propulsion implies repetitive engine firings without earth-based inspection and repair, coating durability will be closely examined, and testing regimes will include repetitive thermal cycling to simulate damage accumulation. The target accomplishments include:

a. Generation of actual hydrogen permeation data for metallic, ceramic-metallic, and hybrid metallic/ceramic composition barrier systems, practically none of which is currently extant.

b. Definition of physical damage modes imported to barrier systems due to thermal cycling, both transient temperature profiles and steady-state thermal mismatch stress states being examined as sources of damage.

c. Computional models that incorporate general laminate schemes as described above, including manufacturing realities such as porosity, and whatever defects are introduced through service and characterized during the experimental programs. 


\section{Current Status and Results}

Efforts to date have been directed towards the following three areas:

\section{A. Laminate system modelling}

Before designing and engaging in relatively complex physical determination of the degree of hydrogen permeation in laminate systems, numerical models were developed to ascertain order-of-magnitude throughput of hydrogen gas. Approximate answers can only be calculated at this time, because permeation data is only available for selected materials, and then often only for bulk samples, not typical of films and coatings. For the case of solid and porous ceramic films, for example, there are orders of magnitude greater ingress (and egress) of hydrogen into Inconel 718 , as can be seen in the following figure.

B. Thermodynamic's of Stress States and Permeation

Recent theoretical developments in thermodynamics hypothesize that permeation of hydrogen is proportional to the hydrostatic, or mean, component of normal stress. However, experimental characterization of material response parameters in this regard are sparse, and do not pertain to relevant materials where they are extant. At the same time, even uniform temperature distributions will lead to residual stress generation in laminate systems, due to thermal mismatch of layer constituents. Coarse extrapolation of marginally relevant data on stress dependence of permeation, matched to realistic levels of stress generated by typical service temperature ranges, strongly suggests that permeation can be measurably altered in real proposed material systems.

\section{Experimental Design}

Parts (A) and (B), above, have driven the particulars of a permeation test cell design that is complete and being fabricated at the time of this writing. At the extreme of laminate effectiveness represented by fully dense ceramic barrier coatings (hardly realizable in practice, but approachable to some degree), downstream vacua levels of $10^{-9}$ Torr were indicated. Accordingly, the permeation cell design was necessarily of the ultrahigh vacuum type (UHV), and a mass spectrometer was deemed necessary to measure the (possibly) slight levels of hydrogen egressing from system samples during the transient phase.

In-situ heating of composite systems was dictated by surface cleanliness considerations whenever temperature effects were to be examined. Temperature was deemed crucial both for residual stress generation as well as fetigue damage generation during service cycling. A quartz lamp heat source and light wire assembly, with suitable UHV transparent porting was designed into the permeation cell system.

At the present time, the cell is being assembled in Room 22 of the Research Building East, the Center headquarters. 


\section{Proposed Work for Coming Year}

A. Experimental facilities will be completed by midsummer, 1990. Completion implies that the UHV hardware will be assembled and tested successfully, including all instrumentation. Two test cells are being manufactured, and their incorporation is expected by early August.

B. Composite material system fabrication will be begun in July as well. This phase of the project will be ongoing for the entire project duration. Various coating technologies will be evaluated to provide metallic, ceramic, and composite coatings.

C. Experimental testing will begin towards the end of August. Several goals will drive the scheduling of the experimental program.

1. Permeation data for many of the candidate coating materials does not exist and will have to be established. In addition, the physical structure of coatings is often vastly different from that associated with monolithic samples of the same chemical composition. Accordingly, permeation data will be obtained for coating microstates that are relevant and related to the particulars of the achievable microstates.

2. Downstream hydrogen concentrations in a given coating will determine availability of the gas to the next constituent in the laminate. That, and the particulars of the laminate interface, will strongly affect multi laminate permeation and this type of system study will be necessary for input into design modelling schemes.

3. Designed residual stress states will be engineered into laminate systems by controlling the choice of thermal response of constituents, by controlling fabrication time/temperature histories, and, finally, by specification of testing (service simulation) temperature.

4. Finally, studies will be undertaken to assess the damage imparted by the thermal cycling that is endemic to space propulsion engine cycling.

It is anticipated that items 1 through 4 will progress throughout the entire year. Although substantial progress towards completion should be accomplished during that time interval, it is anticipated that followup project funding from outside sources will be required to finish even these limited objectives. 
